\title{
Novel Cooling Strategy for Electronic Packages: Directly Injected Cooling
}

\author{
W.W. Wits ${ }^{1}$, G.J.H.M. Brok ${ }^{1}$, J.H. Mannak ${ }^{2}$ and R.Legtenberg ${ }^{2}$ \\ ${ }^{1}$ Laboratory of Design Production and Management, Faculty of Engineering Technology, \\ University of Twente, Enschede, The Netherlands \\ ${ }^{2}$ Thales Netherlands, Hengelo, The Netherlands
}

\begin{abstract}
This publication discusses domain integration of various engineering disciplines as an effective methodology to design new and innovative products. A case study illustrates how this approach is applied to the design process of a high performance electronic product. A novel and improved method for the cooling of electronic packages is presented. Standard package types, as for instance ball grid arrays, are equipped with directly injected cooling. The developed design is a very cost effective solution, as fewer productions steps and fewer procured parts are required compared to traditional cooling. The new design is also easily scalable, as multiple components on an electronic product can be cooled both uniformly and simultaneously. This allows for more overall design flexibility, which can result in a more integrated product design with advantages in terms of performance, volume, weight and production efficiency.
\end{abstract}

\section{Keywords:}

Thermal management, Electronic packages, PCB cooling, Jet impingement cooling

\section{INTRODUCTION}

Thermal management becomes more and more the challenge area in electronic product design. The demand for more functionality and performance, as well as product miniaturization continuously increases. Local heat fluxes are intensified by ever greater power dissipation on smaller surfaces. To effectively manage these increasing heat fluxes, a proper thermal design is required.

This study focuses on developing new cooling strategies for electronic packages. Figure 1 shows a general view of such an electronic package. The heart of the package is formed by the semiconductor die or integrated circuit. Here, all active electronic processes take place, and thus all heat is also generated here. Packaging requirements make it often impossible to attach the die directly to a printed circuit board (PCB); therefore a rigid laminate is used in between. Electronic signals to and from the die run through bondwires, vias in the rigid laminate and the solder balls to the supporting PCB. In this case the ball grid array (BGA) interface is used for both electronic signals and mechanical fixation. Other standardized packaging types as land grid arrays (LGA), pin grid arrays (PGA), stud bump arrays and quad flat-no leads (QFN) are also often seen. Finally, the die is protected from the environment by a mould compound (on component level).

Cooling strategies based on conduction and convection of air are primarily addressed in this study. The authors acknowledge advanced cooling solutions of, for instance, two-phase or on-chip principles. However, as conduction and air convection are relatively straight-forward to implement, we strive to extend the limits of these solutions. The cooling system to be designed should be able to keep an electronic product at a relatively uniform temperature. In addition, a scalable solution is demanded, as high performance electronic products often consist of multiple high power packages.

Instead of focussing only on the thermal issues, the total design is researched in an integrative approach. In-depth and coherent knowledge of every engineering field involved is required to pursue the best system performance at the lowest cost. By designing for all engineering disciplines, a level of integration previously unimaginable is achieved.

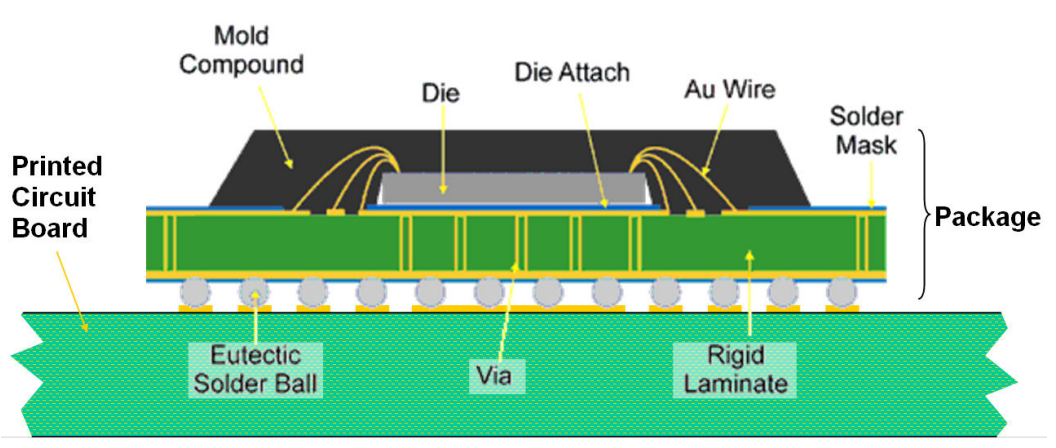

(a) schematic layout

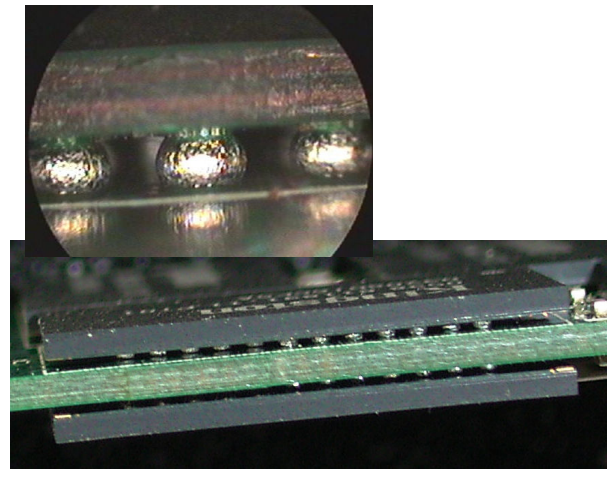

(b) actual product

Figure 1: Ball grid array (BGA) package. 


\section{PACKAGE DESIGN}

Traditionally, the design process of most electronic products has been dominated by electrical and mechanical requirements. As cooling is generally not a primary function of electronic products, thermal analyses were usually addressed towards the end of the design process. This is illustrated by Figure 2 .

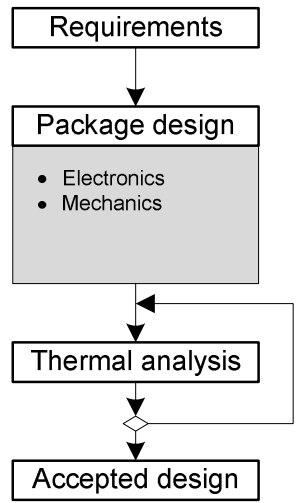

Figure 2: Traditional design process.

In the struggle to keep pace with increasing semiconductor performances, add-on cooling devices are becoming grotesque in size and power consumption, as shown in Figure 3. Although traditional forced convection cooling may have sufficient cooling potential in theory, it is constrained by the fact that the heat dissipating component usually cannot be directly exposed to the cool air. In other words: the generated heat has to travel from the die through a series of thermal resistances before it finally is disposed to the ambient. These thermal resistances consist, for instance, of adhesive layers, encapsulation resins, solder connections, pockets or layers of stationary air and others. This results in a significant and often unacceptable temperature gradient across the die, package and heat sink.

Until recently, thermal management aspects scarcely impeded an optimal product design. However, the state of the art in semiconductor technology has reached a thermal limit. Current package sizes are not in accordance with their required heat sinks. To solve this, a more substantial thermal engineering contribution earlier in the design phase is required.

Many electronic packages dissipate most heat through their top surface to either a heat sink or a more sophisticated (active) cooling device. This has little impact on the PCB design itself. However, this technique has its limitations as in most cases thermal resistance from the semiconductor die to the package top surface is relatively high. Moreover, in some applications the component's top surface is not even accessible, in particular when the electronic package is an integrated sensor. In these cases, most of the heat must be dissipated through the bottom and "footprint" of the package into the PCB. Although in many cases the bottom side of electronic packages has the lowest thermal resistance, this side cannot be directly exposed to a coolant medium because it is facing the PCB. Generally, PCBs have a poor thermal conductivity, which can be moderately improved by adding more or thicker copper layers.

\section{DOMAIN INTEGRATIVE DESIGN}

As indicated in the previous section, due to thermal management issues, a new cooling strategy is required to manage heat dissipations of electronic packages. This is not just an isolated case. In fact, recent research publications indicate that a limit has been reached for cooling electronics in general [1]. As a result, the continuous product improvement cycle threatens to stall, if no appropriate action is taken towards thermal innovation in the design and manufacturing process. The design challenges are conquered through domain integration, resulting in further integration of primary and support functions [2]. Knowledge of heat transfer and production principles are integrated into the overall design process, as illustrated in Figure 4.

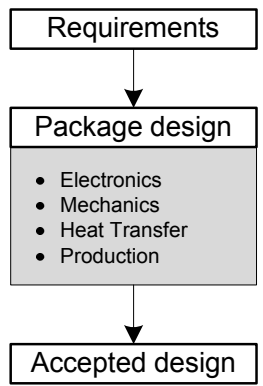

Figure 4: Integrated design process.

Although the design process may seem more compact it actually tends to become more complex due to the addition of thermal and production aspects. A greater number of conflicting relationships needs to be considered, to establish cause-and-effect coherence. The basic strategy is to bring the coolant closer to the heat source and reduce thermal resistance between the heat

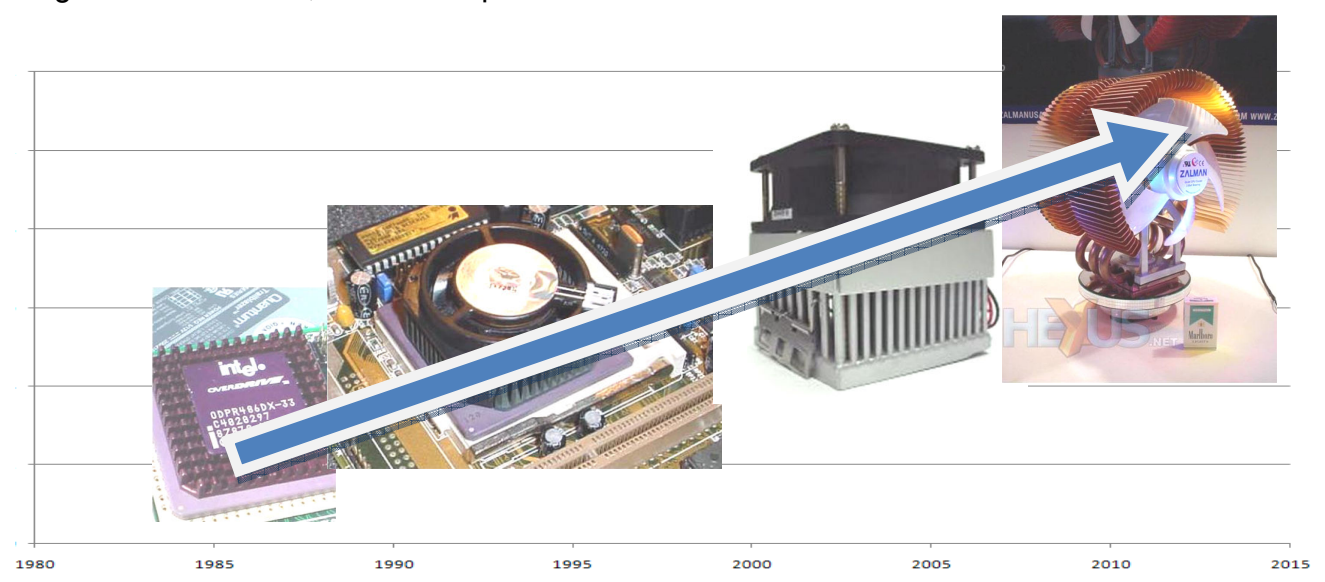

Figure 3: Increasing heat sink sizes. 
source and its heat exchanger. In the case of subject electronic packages the adapted design process has lead to several cooling concepts.

Using a schematic model of the electronic package these concepts are evaluated theoretically. The models show the die (in red) on a rigid laminate. This laminate is connected to the PCB by the BGA. To emphasize the fact that the heat has to be dissipated through the bottom of the package, the die is placed in a box. In the first attempt the heat exchanger is to be placed on the backside of the PCB. To transport the heat efficiently through the board, thermal vias are placed directly below the package, as illustrated in Figure 5(a). Although the thermal vias provide a better conducting path to the heat exchanger, the losses are still significant. Bottlenecks are the BGA interface and the vias; they contribute to up to $90 \%$ of all thermal losses. Increasing the number of vias is also quite expensive.

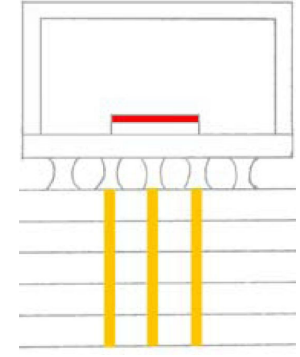

(a) thermal vias

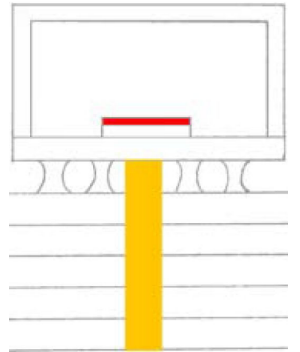

(b) single element
Figure 5: Conduction concepts.

An improved concept is shown in Figure 5(b). Here, heat travels through a single conducting element, instead of through the BGA and the thermal vias. From a thermal point-of-view this concept is acceptable, however in practice this is hard to manufacture. The conducting element has to be positioned against the package with enough preload to obtain a proper thermal connection.

In an attempt to improve manufacturability, the coolant is brought closer to the heat source: the heat exchanger is placed inside the board, as shown in Figure 6(a). Conditioned air can be pumped through a cavity directly below the package. As no external heat sink is required, this saves assembly steps. However, additional assembly steps are necessary to supply each cavity with air. Especially for PCBs with multiple heat sources this is disadvantageous.
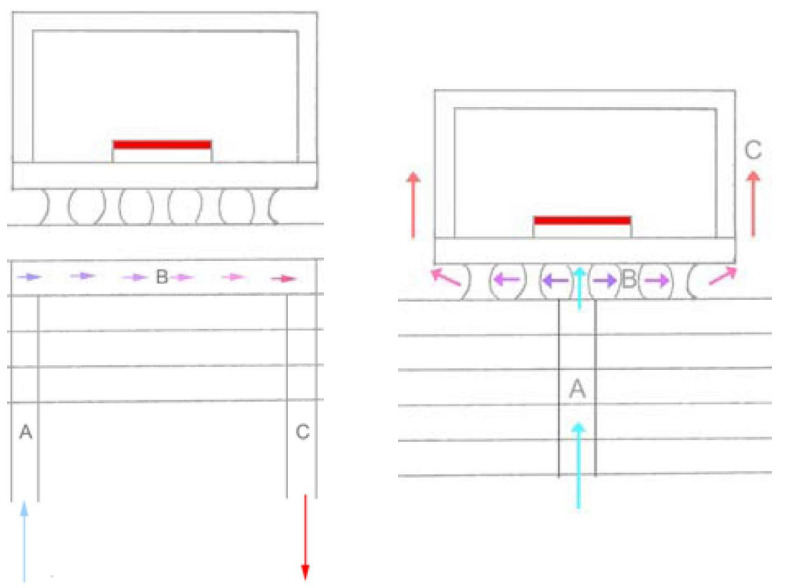

(a) in-board cooling

(b) directly injected cooling
This is resolved in the final concept shown in Figure 6(b). Here, total design integration is reached as the BGA acts as a heat exchanger as well. Conditioned air is pumped from the backside to the front through a hole in the PCB. Instead of connecting a cooling line to each package, the entire PCB can also be placed on a pressure chamber to supply coolant to all heat sources simultaneously.

This concept, for which a patent is pending, is expected to work well for all sorts of electronic packages.

\section{DETAILED DESIGN}

In this novel cooling concept the BGA is not only used for the transmission of electronic signals and mechanical fixation, but also acts as an integrated heat exchanger. For BGA packages the standoff distance is determined by the size of the solder balls and the amount of solder required to make a reliable electrical and mechanical connection. Now, also sufficient height must be created for the coolant medium to pass through, absorbing as much power as required.

By injecting air directly into the BGA, it is brought very close to the hot underside of the rigid laminate, thus bypassing several thermal resistances. In addition, as the cool air approaches the hot surface from a perpendicular direction a jet impingement effect will also occur. This increases the heat transfer rate compared to tangent flow by locally breaking through the thermal boundary layer. Finally, as a BGA typically has a large number of solder connections, the heat exchange area is relatively large. It also promotes turbulent flow, which improves heat transfer even further. In an advanced set-up the balls could even be placed in such a way to maximize cooling potential.

Important parameters for confined jet impingement, according to Glynn [3], are the Reynolds number of the air flow and the ratio of jet-to-target spacing and jet diameter. For optimal heat transfer, this ratio should be low, close to unity:

$$
\frac{h}{d} \approx 1
$$

The jet-to-target spacing $(h)$ is determined by the standoff distance of the rigid laminate. For BGA packages this is typically 0.5 to $1 \mathrm{~mm}$. The jet diameter $(d)$ is determined by the size of the hole in the PCB. According to Equation (1) the diameter should approximately equal the standoff distance. Also to optimize heat transfer, according to Glynn, a high Reynolds number, and thus high air flow velocity should be used. However, in practice this is limited by both the maximum pressure the system can endure and pumping capacity.

Airflow properties to optimize heat transfer are still subject of further investigation. However, based on the literature used, a local heat transfer coefficient of at least $1500 \mathrm{~W} / \mathrm{m}^{2} \mathrm{~K}$ should be feasible. Combining this value with the thermal conduction properties of a typical BGA package, with $1 \mathrm{~cm}^{2}$ die, an overall thermal resistance of junction to ambient as low as $8 \mathrm{KM}$ can be obtained. Here, thermal resistance is defined as:

$$
R_{\text {th }}=\frac{\Delta T_{\text {junction,ambient }}}{P_{\text {dissipated }}} \rightarrow R_{\text {th }} \approx 8 \frac{K}{W}
$$

Therefore, with air conditioned at $25^{\circ} \mathrm{C}$ an electronic package dissipating as much as $15.6 \mathrm{~W}$ can be cooled effectively. It must be noted that this is a preliminary estimate.

Figure 6: Novel concepts. 
During assembly fewer production steps are required, as no external heat sink is required. The only dedicated feature is a (non-plated through) hole in the PCB, which can be produced using standard $\mathrm{PCB}$ production techniques. Heat from multiple sources can also be transported without introducing unacceptable temperature gradients across the PCB, as each heat source has an individual cooling support, as shown in Figure 7. This allows for less constrained and scalable design solutions.

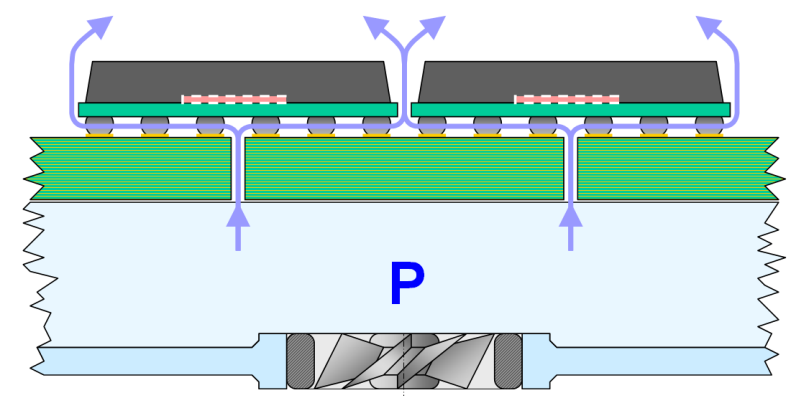

Figure 7: Design for multiple packages.

\section{TECHNOLOGY DEMONSTRATOR}

To prove the concept of this new cooling technique, a technology demonstrator was developed. A test board with various components, each with individual cooling support, was placed on a pressure chamber. The components consisted of a copper laminate with a diode, acting as a power source, mounted onto its surface. Power, and thus heat, was dissipated through the diode, while air was fed to the pressure chamber. Both air pressure and velocity, as well as temperature were measured continuously.

As the air pressure, and thus air velocity, was increased, heat transfer improved. Therefore, more power could be dissipated at lower junction temperatures, as shown in Figure 8. Here, the top line indicates no air flow; at the bottom line the Reynolds number equals 1424 .

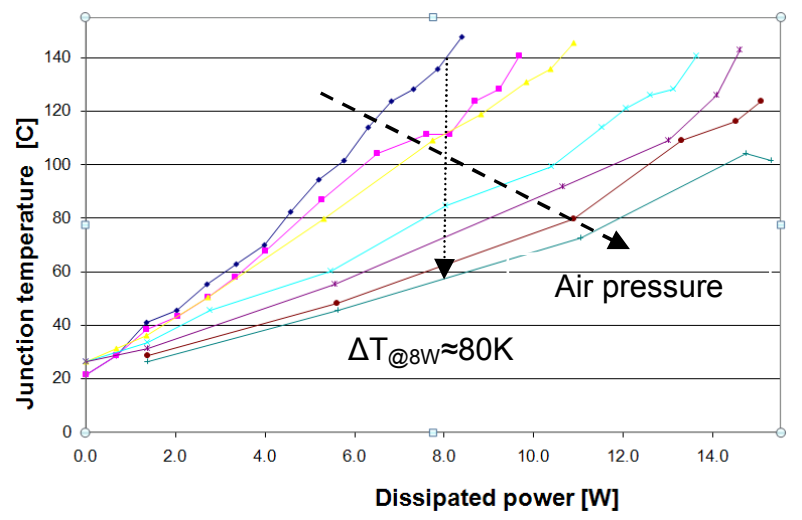

Figure 8: Temperature vs. power measurements.

Measurement results show that the concept of directly injected cooling through a BGA works well. For instance, at a power dissipation of $8 \mathrm{~W}$ a temperature drop of $80 \mathrm{~K}$ was obtained. Also, measurements at higher ratings converge towards a value close to $15.6 \mathrm{~W}$, as calculated previously.
Figure 9 shows a thermal image of one component dissipating $14 \mathrm{~W}$. The die, shown in red, conducts its heat to the rigid laminate (green). On the bottom side the heat is disposed by cool air that passes through the BGA.

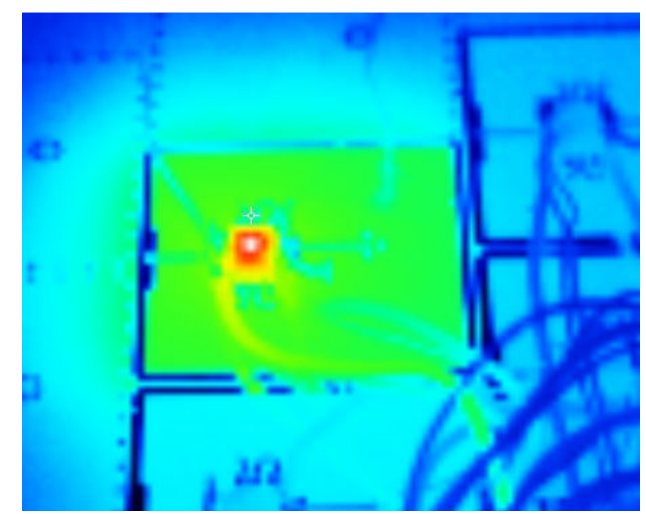

Figure 9: Thermal image of electronic package.

For PCB technology in general, this concept leads to new manufacturing strategies where thermal management, electronics and mechanics are fully integrated, resulting in more compact electronic systems at a lower cost.

\section{CONCLUSIONS}

Through a case study new thermal management and manufacturing strategies for electronic products have been presented. By incorporating thermal and production engineering aspects at an early stage in the design process, more integrated solutions can be realized. Without additional heat sinks and by adding just one (non-plated through) hole in the PCB an electronic package can be cooled efficiently. The new design is also easily scalable, electronic products with multiple components can be cooled both uniformly and simultaneously. This increases design freedom and enables a more multifunctional product design.

Full integration of thermal management functions and electronic circuitry in electronic products pushes the boundary further towards more functionality and performance in a smaller form factor. In addition, products can be realized at a lower cost. Altogether, this can lead to smaller, lighter and more affordable products.

\section{ACKNOWLEDGMENTS}

This work is part of the PACMAN project which is supported by the Netherlands Ministry of Economic Affairs, SenterNovem project number TSIT3049. The authors especially want to thank Jan-Egbert Annevelink and Ton Polman for both their advice and assistance during proof-of-principle research efforts. We also thank the PCB production and electronics assembly department of Thales Nederland for their work on the prototype.

\section{REFERENCES}

[1] Lasance, C.J.M. and Simons, R.E., 2005, Advances in high-performance cooling for electronics, ElectronicsCooling, Volume 11, Number 4.

[2] Suh, N.P., 1990, The principles of design, Oxford University Press.

[3] Glynn, C., O'Donovan, T.S. and Murray, D.B., 2005, Jet Impingement Cooling, Proceedings of the $9^{\text {th }}$ UK National Heat Transfer Conference, Manchester. 